\title{
Balance studies in malnourished Jamaican infants
}

\section{2.* Comparison of absorption and retention of nitrogen and phosphorus from human milk and a cow's-milk mixture}

\author{
BY J. C. WATERLOW AND VERITY G. WILLS \\ Medical Research Council Tropical Metabolism Research Unit, \\ University College of the West Indies, Famaica \\ AND P. GYÖRGY \\ Department of Pediatrics, Philadelphia General Hospital, Philadelphia, Pa. \\ (Received 14 Fuly 1959-Revised 20 October 1959)
}

As part of the work on retention of nitrogen and phosphorus described in the previous paper (Waterlow \& Wills, 1960) a comparison has been made between breast milk and cow's-milk mixtures. Earlier studies of this kind have been done on normal full-term infants aged about I month (Barness, Baker, Guilbert, Torres \& György, 1957) and on premature infants (Gordon, Levine, Wheatley \& Marples, 1937), but significant differences in $\mathrm{N}$ absorption and retention were not found. It seemed worth while to re-investigate this problem, since the malnourished baby's great avidity for protein should make it a sensitive test subject, in whom it may be easier to demonstrate a difference in biological value between the two milks, if one exists.

\section{EXPERIMENTAL}

The seventeen infants studied and the methods used are described in the previous paper (Waterlow \& Wills, I960).

Expressed breast milk (EBM) was collected from mothers in all stages of lactation after the rst week, pooled, and stored in the deep freeze. Two cow's-milk mixtures were used (S.M.A. preparations kindly supplied by Wyeth Laboratories Inc., Philadelphia, U.S.A.), in which the protein content and calorie value were adjusted to equal those of human milk. These mixtures were based on dried skim milk, with the addition of vegetable oil, and either lactose (mixture S I4) or dextrimaltose (mixture $S 20$ ), to give a final composition on reconstitution of protein $I \cdot I$, fat 3.68 , and carbohydrate $6.8 \%$. Twelve of the seventeen babies received the dextrimaltose mixture and three the lactose mixture. For the remaining two by error no record was kept of which mixture was given. There seemed to be no obvious difference between the two $\mathrm{S}$ mixtures, and since the number of subjects was small the results have been analysed together.

In any comparison of this kind it is desirable that the $N$ intakes should be as nearly equal as possible. The mean intakes on EBM and $S$ were very nearly the same

* Paper no. I : Brit. F. Nutr. (1960), 14, 183. 
(Table 2). Out of forty-six balances, there were only five in which the intakes fell outside the range $0.25-0.5^{\circ} \mathrm{g} \mathrm{N} / \mathrm{kg}$ body-weight/day. The findings presented in the previous paper suggest that $\mathrm{N}$ retention increases linearly with intake up to an intake of at least $0.5 \mathrm{~g} / \mathrm{kg} /$ day. Therefore it is unlikely that slight differences in intake within this range would affect the validity of the comparison.

\section{RESULTS}

\section{Nitrogen absorption and retention}

The results are shown in detail in Table $\mathrm{I}$, and in summary in Table 2. The fact that $\mathrm{N}$ retention tends to fall off as treatment progresses (Waterlow \& Wills, 1960) introduces a difficulty in analysing experiments of this kind. In Table 2 the balance periods on each milk have been divided into two groups, according to whether they fell in the first or the second 20 days in hospital.

With both types of milk, $\mathrm{N}$ absorption improved as treatment progressed. In both periods absorption seemed better with EBM than with $\mathrm{S}$, but the differences were not significant.

With both types, $\mathrm{N}$ retention decreased with increased duration of stay in hospital. In both periods retention was better with EBM than with S, but only in the second period was the difference significant.

When the results for the two periods were combined, and all EBM results were compared with all $S$ results, retention was significantly better with EBM.

This analysis ignores the fact that each subject serves as its own control. A further comparison has therefore been made to take account both of this feature and of the time factor. In fourteen infants an EBM balance preceded an S balance (group A), and in thirteen infants an EBM balance followed an $\mathrm{S}$ balance (group B). As will be seen from Table I, several infants were common to both groups.

For each pair of balances a figure was obtained for the difference in percentage absorption or retention between the two milks. The results are shown in Table 3. If there was no difference between the milks, EBM would have an advantage in group A, where it preceded $S$, but this advantage should be reversed in group $B$, where it followed $\mathrm{S}$. In fact, $\mathrm{N}$ retention was significantly better with $\mathrm{EBM}$ in both groups. It is particularly striking that it remained better when EBM followed S (group B). This superiority was partly, but not entirely, due to better absorption.

The $P$ content of breast milk (about $150 \mathrm{mg} / \mathrm{l}$.) is only half that of the $\mathrm{S}$ mixtures (300 $\mathrm{mg} / \mathrm{l}$.). Since we had found that these depleted babies retain even more $P$ than $\mathrm{N}$, by comparison with the $\mathrm{P}$ and $\mathrm{N}$ content of normal soft tissues, it seemed possible that the low $\mathrm{P}$ content of breast milk might be a limiting factor, reducing the rate of $\mathrm{N}$ retention. Therefore for three infants (Table I, nos. 14-16) balances were done with breast milk to which $\mathrm{P}$ was added (as sodium-phosphate solution, $\mathrm{pH} 7 \cdot 4$ ) to bring the $\mathrm{P}$ content up to that of mixture $\mathrm{S}$. As Table I shows, there was no improvement in $\mathrm{N}$ retention. 
Table I. Absorption and retention of nitrogen and phosphorus in malnourished infants on breast milk (EBM) and on a cow's-milk mixture ( $S_{14}$ or $\left.S_{20}\right)$

\begin{tabular}{|c|c|c|c|c|c|c|c|c|c|c|c|}
\hline \multirow[b]{3}{*}{$\begin{array}{l}\text { Subject } \\
\text { no. }\end{array}$} & \multirow[b]{3}{*}{$\begin{array}{c}\text { Age } \\
\text { (mths) }\end{array}$} & \multirow{3}{*}{$\begin{array}{l}\text { Period after } \\
\text { admission } \\
\text { (days) }\end{array}$} & \multirow[b]{3}{*}{ Diet } & \multicolumn{4}{|c|}{ Nitrogen } & \multicolumn{4}{|c|}{ Phosphorus } \\
\hline & & & & \multirow{2}{*}{$\begin{array}{c}\text { Intake } \\
\text { (g/kg } \\
\text { body-wt } / \\
\text { day) }\end{array}$} & \multirow{2}{*}{$\begin{array}{l}\text { Absorp- } \\
\text { tion } \\
\text { (as \% of } \\
\text { intake) }\end{array}$} & \multicolumn{2}{|c|}{$\begin{array}{c}\text { Retention } \\
\text { as } \% \text { of }\end{array}$} & \multirow{2}{*}{$\begin{array}{c}\text { Intake } \\
\text { (mg/kg } \\
\text { body-wt/ } \\
\text { day) }\end{array}$} & \multirow{2}{*}{$\begin{array}{l}\text { Absorp- } \\
\text { tion } \\
\text { (as \% of } \\
\text { intake) }\end{array}$} & \multicolumn{2}{|c|}{$\begin{array}{l}\text { Retention } \\
\text { as } \% \text { of }\end{array}$} \\
\hline & & & & & & Intake & $\begin{array}{c}\text { Absorp- } \\
\text { tion }\end{array}$ & & & Intake & $\begin{array}{c}\text { Absorp- } \\
\text { tion }\end{array}$ \\
\hline $\mathbf{I}$ & 16 & $\begin{array}{l}13-19 \\
21-26\end{array}$ & $\begin{array}{l}\text { EBM } \\
S^{*}\end{array}$ & $\begin{array}{l}0.39 \\
0.37\end{array}$ & $\begin{array}{l}83 \\
89\end{array}$ & $\begin{array}{l}42 \\
45\end{array}$ & $\begin{array}{l}51 \\
50\end{array}$ & $\begin{array}{l}32 \\
59\end{array}$ & $\begin{array}{l}69 \\
96\end{array}$ & $\begin{array}{l}55 \\
45\end{array}$ & $\begin{array}{l}79 \\
47\end{array}$ \\
\hline 2 & 16 & $\begin{array}{l}14-16 \\
17-23 \\
31-33\end{array}$ & $\begin{array}{l}S^{*} \\
\text { EBM } \\
S^{*}\end{array}$ & $\begin{array}{l}0.30 \\
0.29 \\
0.29\end{array}$ & $\begin{array}{l}83 \\
90 \\
84\end{array}$ & $\begin{array}{l}37 \\
40 \\
24\end{array}$ & $\begin{array}{l}45 \\
43 \\
29\end{array}$ & $\begin{array}{l}54 \\
20 \\
52\end{array}$ & $\begin{array}{l}80 \\
71 \\
58\end{array}$ & $\begin{array}{l}34 \\
55 \\
16\end{array}$ & $\begin{array}{l}42 \\
77 \\
27\end{array}$ \\
\hline 3 & I I & $\begin{array}{r}7-12 \\
19-24\end{array}$ & $\begin{array}{l}\text { S I4 } \\
\text { EBM }\end{array}$ & $\begin{array}{l}0.33 \\
0.32\end{array}$ & $\begin{array}{l}80 \\
86\end{array}$ & $\begin{array}{l}24 \\
34\end{array}$ & $\begin{array}{l}30 \\
39\end{array}$ & $\begin{array}{l}60 \\
26\end{array}$ & $\begin{array}{l}76 \\
76\end{array}$ & $\begin{array}{l}22 \\
53\end{array}$ & $\begin{array}{l}29 \\
70\end{array}$ \\
\hline 4 & 15 & $\begin{array}{c}0-8 \\
10-15 \\
18-20\end{array}$ & $\begin{array}{l}\text { S } 20 \\
\text { EBM } \\
\text { S } 20\end{array}$ & $\begin{array}{l}0.32 \\
0.39 \\
0.39\end{array}$ & $\begin{array}{l}75 \\
84 \\
79\end{array}$ & $\begin{array}{l}48 \\
60 \\
42\end{array}$ & $\begin{array}{l}64 \\
72 \\
5^{2}\end{array}$ & $\begin{array}{l}57 \\
28 \\
70\end{array}$ & $\begin{array}{l}70 \\
76 \\
59\end{array}$ & $\begin{array}{l}30 \\
55 \\
18\end{array}$ & $\begin{array}{l}42 \\
73 \\
30\end{array}$ \\
\hline 5 & 12 & $\begin{array}{r}8-17 \\
19-25 \\
26-37 \\
39-47\end{array}$ & $\begin{array}{l}S_{14} \\
\text { EBM } \\
S_{14} \\
S_{20}\end{array}$ & $\begin{array}{l}0.36 \\
0.30 \\
0.40 \\
0.42\end{array}$ & $\begin{array}{l}76 \\
88 \\
87 \\
90\end{array}$ & $\begin{array}{l}43 \\
47 \\
46 \\
50\end{array}$ & $\begin{array}{l}57 \\
54 \\
52 \\
56\end{array}$ & $\begin{array}{l}64 \\
32 \\
70 \\
73\end{array}$ & $\begin{array}{l}61 \\
82 \\
75 \\
82\end{array}$ & $\begin{array}{l}27 \\
71 \\
31 \\
37\end{array}$ & $\begin{array}{l}44 \\
87 \\
41 \\
45\end{array}$ \\
\hline 6 & 15 & $\begin{array}{r}8-13 \\
14-24 \\
25-33\end{array}$ & $\begin{array}{l}\text { S 20 } \\
\text { EBM } \\
\text { S 20 }\end{array}$ & $\begin{array}{l}0.39 \\
0.43 \\
0.39\end{array}$ & $\begin{array}{l}70 \\
74 \\
79\end{array}$ & $\begin{array}{l}42 \\
47 \\
44\end{array}$ & $\begin{array}{l}60 \\
63 \\
55\end{array}$ & $\begin{array}{l}71 \\
32 \\
73\end{array}$ & $\begin{array}{l}54 \\
58 \\
69\end{array}$ & $\begin{array}{l}24 \\
56 \\
30\end{array}$ & $\begin{array}{l}44 \\
98 \\
43\end{array}$ \\
\hline 7 & 15 & $\begin{array}{r}8-13 \\
14-24 \\
25-33\end{array}$ & $\begin{array}{l}\text { S 20 } \\
\text { EBM } \\
S \text { 20 }\end{array}$ & $\begin{array}{l}0.44 \\
0.42 \\
0.42\end{array}$ & $\begin{array}{l}72 \\
76 \\
89\end{array}$ & $\begin{array}{l}49 \\
52 \\
44\end{array}$ & $\begin{array}{l}68 \\
68 \\
52\end{array}$ & $\begin{array}{l}80 \\
32 \\
74\end{array}$ & $\begin{array}{l}78 \\
64 \\
80\end{array}$ & $\begin{array}{l}35 \\
57 \\
41\end{array}$ & $\begin{array}{l}44 \\
90 \\
51\end{array}$ \\
\hline 8 & I7 & $\begin{array}{l}23-3 I \\
33-38\end{array}$ & $\begin{array}{l}\mathrm{EBM} \\
\mathrm{S}_{20}\end{array}$ & $\begin{array}{l}0.31 \\
0.32\end{array}$ & $\begin{array}{l}82 \\
82\end{array}$ & $\begin{array}{l}47 \\
39\end{array}$ & $\begin{array}{l}57 \\
4^{8}\end{array}$ & $\begin{array}{l}24 \\
59\end{array}$ & $\begin{array}{l}72 \\
80\end{array}$ & $\begin{array}{l}71 \\
12\end{array}$ & $\begin{array}{l}99 \\
15\end{array}$ \\
\hline 9 & 22 & $\begin{array}{l}10-20 \\
21-29\end{array}$ & $\begin{array}{l}\text { EBM } \\
S_{20}\end{array}$ & $\begin{array}{l}0.34 \\
0.38\end{array}$ & $\begin{array}{l}79 \\
88\end{array}$ & $\begin{array}{l}39 \\
40\end{array}$ & $\begin{array}{l}49 \\
46\end{array}$ & $\begin{array}{l}24 \\
67\end{array}$ & $\begin{array}{l}52 \\
80\end{array}$ & $\begin{array}{l}52 \\
34\end{array}$ & $\begin{array}{r}100 \\
43\end{array}$ \\
\hline Io & 15 & $\begin{array}{r}0-10 \\
12-20 \\
21-29 \\
30-38\end{array}$ & $\begin{array}{l}\text { S } 20 \\
\text { EBM } \\
\text { S } 20 \\
\text { EBM }\end{array}$ & $\begin{array}{l}0.22 \\
0.28 \\
0.34 \\
0.31\end{array}$ & $\begin{array}{l}81 \\
78 \\
81 \\
85\end{array}$ & $\begin{array}{l}46 \\
54 \\
49 \\
44\end{array}$ & $\begin{array}{l}58 \\
70 \\
60 \\
5 x\end{array}$ & $\begin{array}{l}36 \\
20 \\
60 \\
22\end{array}$ & $\begin{array}{l}61 \\
57 \\
75 \\
69\end{array}$ & $\begin{array}{l}36 \\
24 \\
25 \\
40\end{array}$ & $\begin{array}{l}59 \\
42 \\
33 \\
58\end{array}$ \\
\hline 11 & 3 & $\begin{array}{r}0-11 \\
13-21 \\
22-30\end{array}$ & $\begin{array}{l}\text { EBM } \\
\text { S 20 } \\
\text { EBM }\end{array}$ & $\begin{array}{l}0.49 \\
0.52 \\
0.46\end{array}$ & $\begin{array}{l}80 \\
79 \\
82\end{array}$ & $\begin{array}{l}56 \\
49 \\
51\end{array}$ & $\begin{array}{l}70 \\
62 \\
62\end{array}$ & $\begin{array}{l}38 \\
89 \\
35\end{array}$ & $\begin{array}{l}81 \\
82 \\
80\end{array}$ & $\begin{array}{l}70 \\
14 \\
58\end{array}$ & $\begin{array}{l}86 \\
17 \\
73\end{array}$ \\
\hline 12 & 9 & $\begin{array}{l}16-24 \\
31-33\end{array}$ & $\begin{array}{l}\text { S 20 } \\
\text { EBM }\end{array}$ & $\begin{array}{l}0.41 \\
0.45\end{array}$ & $\begin{array}{l}82 \\
92\end{array}$ & $\begin{array}{l}56 \\
70\end{array}$ & $\begin{array}{l}68 \\
76\end{array}$ & $\begin{array}{l}72 \\
30\end{array}$ & $\begin{array}{l}83 \\
92\end{array}$ & $\begin{array}{l}36 \\
60\end{array}$ & $\begin{array}{l}44 \\
66\end{array}$ \\
\hline I3 & 13 & $\begin{array}{l}14-26 \\
27-36 \\
37-45\end{array}$ & $\begin{array}{l}\text { S } 20 \\
\text { EBM } \\
\text { S } 20\end{array}$ & $\begin{array}{l}0.42 \\
0.47 \\
0.38\end{array}$ & $\begin{array}{l}78 \\
79 \\
66\end{array}$ & $\begin{array}{l}50 \\
53 \\
22\end{array}$ & $\begin{array}{l}64 \\
67 \\
34\end{array}$ & $\begin{array}{l}73 \\
33 \\
70\end{array}$ & $\begin{array}{l}76 \\
59 \\
64\end{array}$ & $\begin{array}{r}16 \\
32 \\
6\end{array}$ & $\begin{array}{r}20 \\
54 \\
8\end{array}$ \\
\hline 14 & 17 & $\begin{array}{l}24-35 \\
37-44 \\
45-5 x \\
55-65\end{array}$ & $\begin{array}{l}\text { S 20 } \\
\text { EBM } \\
\text { EBM } \dagger \\
\text { S } 20\end{array}$ & $\begin{array}{l}0.40 \\
0.42 \\
0.44 \\
0.32\end{array}$ & $\begin{array}{l}72 \\
80 \\
79 \\
73\end{array}$ & $\begin{array}{l}26 \\
38 \\
39 \\
31\end{array}$ & $\begin{array}{l}36 \\
48 \\
50 \\
42\end{array}$ & $\begin{array}{l}70 \\
30 \\
65 \\
58\end{array}$ & $\begin{array}{l}61 \\
47 \\
74 \\
67\end{array}$ & $\begin{array}{l}12 \\
18 \\
24 \\
21\end{array}$ & $\begin{array}{l}20 \\
38 \\
32 \\
32\end{array}$ \\
\hline 15 & 4 & $\begin{array}{l}10-21 \\
23-30 \\
30-38\end{array}$ & $\begin{array}{l}\text { S } 20 \\
\text { EBM } \\
\text { EBM } \dagger\end{array}$ & $\begin{array}{l}0.43 \\
0.51 \\
0.46\end{array}$ & $\begin{array}{l}77 \\
80 \\
73\end{array}$ & $\begin{array}{l}45 \\
58 \\
50\end{array}$ & $\begin{array}{l}58 \\
73 \\
69\end{array}$ & $\begin{array}{l}76 \\
40 \\
67\end{array}$ & $\begin{array}{l}75 \\
72 \\
74\end{array}$ & $\begin{array}{l}34 \\
72 \\
48\end{array}$ & $\begin{array}{r}46 \\
100 \\
65\end{array}$ \\
\hline 16 & 7 & $\begin{array}{l}24-29 \\
30-35 \\
38-49\end{array}$ & $\begin{array}{l}\text { EBM } \\
\text { EBM } † \\
\text { S } 20\end{array}$ & $\begin{array}{l}0.24 \\
0.30 \\
0.31\end{array}$ & $\begin{array}{l}90 \\
76 \\
76\end{array}$ & $\begin{array}{l}48 \\
38 \\
30\end{array}$ & $\begin{array}{l}54 \\
50 \\
40\end{array}$ & $\begin{array}{l}17 \\
45 \\
42\end{array}$ & $\begin{array}{l}83 \\
81 \\
71\end{array}$ & $\begin{array}{c}80 \\
27 \\
\text { Neg. }\end{array}$ & $\begin{array}{c}97 \\
33 \\
\text { Neg. }\end{array}$ \\
\hline 17 & 5 & $\begin{array}{r}8-17 \\
22-33\end{array}$ & $\begin{array}{l}\text { EBM } \\
S_{14}\end{array}$ & $\begin{array}{l}0.24 \\
0.28\end{array}$ & $\begin{array}{l}78 \\
74\end{array}$ & $\begin{array}{l}50 \\
39\end{array}$ & $\begin{array}{l}64 \\
53\end{array}$ & $\begin{array}{l}22 \\
54\end{array}$ & $\begin{array}{l}84 \\
75\end{array}$ & $\begin{array}{l}72 \\
23\end{array}$ & $\begin{array}{l}86 \\
30\end{array}$ \\
\hline
\end{tabular}

Neg., negative. $\quad \mathrm{S}_{4}$ or S 20 (see p. 199). + P added as sodium phosphate (see p. 200). 


\section{Phosphorus absorption and retention}

The results are shown in summary in Table 4 . On both milks, $70 \%$ of the ingested $P$ was absorbed. The $P$ intake on EBM was less than half that on mixture $S$, but the percentage retention was twice as great, so that the absolute amounts retained were almost identical on the two milks.

As was noted by Waterlow \& Wills (1960), the efficiency of P utilization seems to be very great. Some babies on EBM, particularly in the early stages of treatment, retained over $90 \%$ of absorbed $P$.

Table 2. Summary of nitrogen absorption and retention by malnourished infants on breast milk $(E B M)$ or on a cow's-milk mixture $(S)$

(Mean values with their standard errors)

\begin{tabular}{|c|c|c|c|c|c|}
\hline Diet & $\begin{array}{l}\text { No. of } \\
\text { subjects }\end{array}$ & $\begin{array}{l}\mathrm{N} \text { intake } \\
\text { (g/kg body- } \\
\text { weight } / \text { day) }\end{array}$ & $\begin{array}{l}N \text { absorbed } \\
\text { (as percentage } \\
\text { of intake) }\end{array}$ & $\begin{array}{l}\text { N retained } \\
\text { (as percentage } \\
\text { of intake) }\end{array}$ & $\begin{array}{l}\mathrm{N} \text { retained } \\
\text { (as percentage } \\
\text { of } \mathrm{N} \text { absorbed) }\end{array}$ \\
\hline \multicolumn{6}{|c|}{ Stage $x$, first 20 days in hospital } \\
\hline $\begin{array}{l}\text { EBM } \\
\text { S } \\
\text { Significance of difference }\end{array}$ & $\begin{array}{r}9 \\
\text { II } \\
-\end{array}$ & $\begin{array}{l}0.363 \\
0.376 \\
-\end{array}$ & $\begin{array}{l}80 \cdot 4 \pm 2 \cdot 20 \\
77 \cdot 6 \pm 1 \cdot 35 \\
\text { N.S. }\end{array}$ & $\begin{array}{l}48 \cdot 9 \pm 3 \cdot 00 \\
44 \cdot 6 \pm 3 \cdot 95 \\
\text { N.S. }\end{array}$ & $\begin{array}{l}61 \cdot 0 \pm 3 \cdot 80 \\
57.5 \pm 3.55 \\
\text { N.S. }\end{array}$ \\
\hline \multicolumn{6}{|c|}{ Stage 2 , second 20 days in hospital } \\
\hline $\begin{array}{l}\text { EBM } \\
\text { S } \\
\text { Significance of difference }\end{array}$ & $\begin{array}{l}10 \\
12 \\
-\end{array}$ & $\begin{array}{l}0.379 \\
0.357 \\
-\end{array}$ & $\begin{array}{c}84 \cdot 4 \pm 2 \cdot 30 \\
81 \cdot 1 \pm 2 \cdot 40 \\
\text { N.S. }\end{array}$ & $\begin{array}{l}49 \cdot 0 \pm 3 \cdot 20 \\
38 \cdot 4 \pm 2 \cdot 40 \\
0.01<P \\
<0.05\end{array}$ & $\begin{aligned} 57.9 & \pm 3.75 \\
47.75 & \pm 2.35 \\
P & \sim 0.05\end{aligned}$ \\
\hline \multicolumn{6}{|c|}{ Both stages together } \\
\hline $\begin{array}{l}\text { EBM } \\
\text { S } \\
\text { Significance of difference }\end{array}$ & $\begin{array}{l}19 \\
23 \\
-\end{array}$ & $\begin{array}{c}0.371 \\
0.366 \\
-\end{array}$ & $\begin{array}{l}82 \cdot 5 \pm 1 \cdot 57 \\
79 \cdot 4 \pm 1 \cdot 36 \\
\text { N.S. }\end{array}$ & $\begin{array}{r}49.0 \pm 2 \cdot 15 \\
41.0 \pm \pm 1.88 \\
P \sim 0.01\end{array}$ & $\begin{array}{l}59 \cdot 4 \pm 2 \cdot 65 \\
52 \cdot 4 \pm 2 \cdot 3^{8} \\
P \sim 0.05\end{array}$ \\
\hline \multicolumn{6}{|c|}{$\begin{array}{l}\text { N.S., not significant }(P>0.05) \text {. } \\
\text { A balance period was assigned to the first or second stage, according to whether the middle day of the } \\
\text { eriod fell in the first or the second } 20 \text { days after admission to hospital. }\end{array}$} \\
\hline \multicolumn{6}{|c|}{ Weight gain } \\
\hline \multicolumn{6}{|c|}{$\begin{array}{l}\text { Waterlow \& Wills ( } 1960 \text { ) pointed out that in these undernourished babies weight } \\
\text { changes are difficult to interpret during the rst month after admission, because gains } \\
\text { in tissue are masked by loss of water. When weight gains were compared for balances } \\
\text { of which the middle day was } 20 \text { days or more after admission to hospital there was no } \\
\text { significant difference between the two milks. On EBM the mean weight gain was } \\
4.49 \text { and on } S 4.84 \mathrm{~g} / \mathrm{kg} / \text { day. }\end{array}$} \\
\hline
\end{tabular}

\section{DISCUSSION}

The lower $\mathrm{N}$ retention found on the cow's-milk mixtures might be attributed to the fact that for most of the subjects the mixture used ( $\mathrm{S} 20$ ) contained dextrimaltose as the main source of carbohydrate. In an earlier investigation slightly better retention was found with the lactose than with the dextrimaltose mixture (Cornely, Barness \& György, 1957). However, those results were not clear-cut, particularly when account 
is taken of the fact that, even in the normal infants studied, $\mathrm{N}$ retention tended to fall off with consecutive balances, regardless of the mixture that was given. In our work, although the number of subjects was small, $\mathrm{S}_{14}$ (lactose) did not appear to have any advantage over S 20 (dextrimaltose). At present, therefore, it seems to us unlikely that the superiority of breast milk over the cows'-milk mixtures results from a difference in the nature of the carbohydrate, although this point will be investigated.

Table 3. Differences for individual malnourished infants in absorption and retention of nitrogen on breast milk (EBM) and on a cow's-milk mixture $(S)^{*}$

\begin{tabular}{|c|c|c|c|c|}
\hline \multirow[b]{2}{*}{$\begin{array}{c}\text { Subject } \\
\text { no. }\end{array}$} & \multirow[b]{2}{*}{$\begin{array}{l}\text { Balance } \\
\text { periods } \\
\text { compared } \\
\text { (Table I) }\end{array}$} & \multicolumn{3}{|c|}{ Difference $(\mathrm{EBM}-\mathrm{S})$} \\
\hline & & $\begin{array}{l}\text { Percentage } \\
\text { absorption }\end{array}$ & $\begin{array}{l}\text { Percentage } \\
\text { retention }\end{array}$ & $\begin{array}{c}\text { Retention as } \\
\text { percentage o } \\
\text { absorption }\end{array}$ \\
\hline \multicolumn{5}{|c|}{ EBM given before $\mathrm{S}$} \\
\hline 1 & I, 2 & $-6 \cdot 0$ & $-2 \cdot 5$ & +0.5 \\
\hline 2 & 2,3 & +6.0 & +15.0 & +14.0 \\
\hline 4 & 2,3 & +5.0 & +18.5 & +19.5 \\
\hline 5 & 2,3 & $+1 \cdot 0$ & $+I .5$ & $+1 \cdot 5$ \\
\hline 6 & 2,3 & -4.5 & +3.0 & +8.0 \\
\hline 7 & 2,3 & $-12 \cdot 5$ & +7.5 & +15.5 \\
\hline 8 & 1,2 & 0 & $+8 \cdot 0$ & +9.5 \\
\hline 9 & 1,2 & -9.0 & $-1 \cdot 0$ & +3.5 \\
\hline Io & 2,3 & -3.0 & +5.5 & +9.5 \\
\hline II & I, 2 & $+1 \cdot 0$ & +7.0 & +8.0 \\
\hline I3 & 2,3 & +13.5 & +30.5 & $+33 \cdot 0$ \\
\hline 14 & 2,4 & $+7 \cdot 0$ & $+7 \cdot 0$ & +5.5 \\
\hline 16 & 1,3 & +14.0 & $+18 \cdot 0$ & +13.5 \\
\hline 17 & 1,2 & +5.0 & $+11 \cdot 5$ & $+11 \cdot 5$ \\
\hline Mean & & $+1 \cdot 25$ & +9.25 & +10.9 \\
\hline $\begin{array}{l}\text { S.E. } \\
\text { Signific }\end{array}$ & & $\begin{array}{l} \pm 2.00 \\
\text { N.S. }\end{array}$ & $\begin{array}{c} \pm=2.25 \\
=0.00 \mathrm{r}\end{array}$ & $\begin{array}{c} \pm \pm 2.15 \\
P=0.001\end{array}$ \\
\hline
\end{tabular}

\section{EBM given after $\mathrm{S}$}

$\begin{array}{ccccc}2 & 1,2 & +7.0 & +2.5 & -2.0 \\ 3 & 1,2 & +6.5 & +9.5 & +9.0 \\ 4 & 1,2 & +8.5 & +12.0 & +7.5 \\ 5 & 1,2 & +12.0 & +4.0 & -3.5 \\ 6 & 1,2 & +4.5 & +4.5 & +2.5 \\ 7 & 1,2 & +4.5 & +2.5 & 0 \\ 10 & 1,2 & -3.0 & +8.0 & +12.0 \\ 10 & 3,4 & +4.0 & -5.5 & -9.0 \\ 11 & 2,3 & +3.5 & +2.0 & -0.5 \\ 12 & 1,2 & +10.0 & +14.0 & +8.5 \\ 13 & 1,2 & +1.0 & +3.0 & +3.0 \\ 14 & 1,2 & +8.0 & +12.0 & +11.5 \\ 15 & 1,2 & +3.0 & +13.5 & +15.0 \\ \text { Mean } & & +5.35 & +6.00 & +4.15 \\ \text { S.E. } & & \pm 1.05 & \pm 1.47 & \pm 1.88 \\ \text { Significancet } & & P=0.001 & P=0.001 & P=0.05\end{array}$

N.S., not significant.

- Balance periods in which phosphorus was added to breast milk (see Table I) have not been included in this analysis.

$\dagger$ Significance of difference from zero by $t$ test, on the null hypothesis that the two milks were the same. 
There are, of course, many differences between human milk and cow's milk, even when the gross composition has been equalized. There are minor differences in the amino-acid composition of the proteins (Block \& Bolling, 1946), and a large difference in the relative amounts of non-protein nitrogen (Macy, Kelley \& Sloan, 1950). The two milks differ also in their content of $P$, of hexosamine and sialic acid (Bennich, Johansson \& Mellander, I957) and of the bifidus factor (György, I953). Human-milk casein, according to Bennich et al. (1957) is less easily digested in vitro than cow's-milk casein, a fact which may be related to its lower $P$ content. No doubt there are other differences still to be discovered.

Table 4. Summary of phosphorus absorption and retention by malnourished infants on breast milk $(E B M)$ or on a cow's-milk mixture $(S)$

\begin{tabular}{|c|c|c|c|c|c|c|}
\hline Diet & $\begin{array}{l}\text { No. of } \\
\text { subjects }\end{array}$ & $\begin{array}{c}\mathrm{P} \text { intake } \\
(\mathrm{mg} / \mathrm{kg} \text { body- } \\
\text { weight } / \text { day) }\end{array}$ & $\begin{array}{c}P \text { absorbed } \\
\text { (as percentage } \\
\text { of intake) }\end{array}$ & $\begin{array}{c}\mathrm{P} \text { retained } \\
\text { (as percentage } \\
\text { of intake) }\end{array}$ & $\begin{array}{c}\mathrm{P} \text { retained } \\
\text { (as percentage } \\
\text { of } \mathrm{P} \text { absorbed) }\end{array}$ & $\begin{array}{l}\text { P retaine } \\
(\mathrm{mg} / \mathrm{kg} \mathrm{bo} \\
\text { weight } / \mathrm{da}\end{array}$ \\
\hline \multicolumn{7}{|c|}{ Stage 1, first 20 days in hospital } \\
\hline $\begin{array}{l}\text { EBM } \\
\mathbf{S}\end{array}$ & $\begin{array}{r}9 \\
\text { II }\end{array}$ & $\begin{array}{l}27^{\circ} 4 \\
66 \cdot 5\end{array}$ & $\begin{array}{l}68 \cdot 0 \\
72 \cdot 3\end{array}$ & $\begin{array}{l}55 \cdot 1 \\
28 \cdot 0\end{array}$ & $\begin{array}{l}81 \cdot 2 \\
39 \cdot 3\end{array}$ & $\begin{array}{l}\text { I5.3 } \\
\text { I } 8 \cdot 2\end{array}$ \\
\hline \multicolumn{7}{|c|}{ Stage 2, second 20 days in hospital } \\
\hline $\begin{array}{l}\text { EBM } \\
\text { S }\end{array}$ & $\begin{array}{l}10 \\
12\end{array}$ & $\begin{array}{l}28 \cdot 8 \\
62 \cdot 6\end{array}$ & $\begin{array}{l}73 \cdot 1 \\
73 \cdot 9\end{array}$ & $\begin{array}{l}55.5 \\
22.5\end{array}$ & $\begin{array}{l}74 \cdot 1 \\
29 \cdot 3\end{array}$ & $\begin{array}{l}15 \cdot 8 \\
14.7\end{array}$ \\
\hline \multicolumn{7}{|c|}{ Both stages together } \\
\hline $\begin{array}{l}\text { EBM } \\
\text { S }\end{array}$ & $\begin{array}{l}19 \\
23\end{array}$ & $\begin{array}{l}28 \cdot 1 \\
64 \cdot 5\end{array}$ & $\begin{array}{l}70 \cdot 7 \\
73 \cdot 0\end{array}$ & $\begin{array}{l}55 \cdot 3 \\
25 \cdot 1\end{array}$ & $\begin{array}{l}77 \cdot 5 \\
34 \cdot 0\end{array}$ & $\begin{array}{l}\text { I5.5 } \\
\text { I6.3 }\end{array}$ \\
\hline
\end{tabular}

$\mathrm{N}$ retention on human milk was better than on the cow's-milk mixtures by about $17 \%$ (mean retention on EBM, $49 \%$; on $\mathrm{S}, 41 \%$ ). It may be thought that this advantage of human milk is too small to be of any biological importance. It may be so in countries where milk supplies are adequate and infants receive daily $3 \mathrm{~g}$ protein $/ \mathrm{kg}$ body-weight or more; it does not necessarily follow when conditions are less favourable. For example, we have been able to measure the food intake of four babies in their homes while kwashiorkor was developing. Their mean protein intake was $0.49 \mathrm{~g} / \mathrm{kg} /$ day. It is probable that in Jamaica and in other countries many babies exist on protein intakes of $\mathrm{r} \mathrm{g} / \mathrm{kg} /$ day without becoming acutely ill from malnutrition. At this level even slight differences in nutritive value may be important. The effect of this marginal intake is reflected, we believe, in the high mortality rate of children between $I$ and 4 years of age (Wills \& Waterlow, I958), which in these countries is five to ten times higher than in Europe or North America.

In this connexion some recent observations of Gopalan (1958) are of great interest. Six normal infants nearly 6 months old, with a mean weight of $5.5 \mathrm{~kg}$, were gaining weight at the rate of $100 \mathrm{~g} /$ week. If the $\mathrm{N}$ content of the tissue laid down is taken as $2.5 \%$, they must have been retaining about $0.065 \mathrm{~g} \mathrm{~N} / \mathrm{kg} /$ day. Their sole source of protein was $500 \mathrm{ml}$ human milk/day. If it had a $\mathrm{N}$ content of $1 \cdot 7 \mathrm{~g} / \mathrm{l}$, the mean intake would be $0.155 \mathrm{~g} \mathrm{~N}$ (equivalent to $0.97 \mathrm{~g}$ protein) $/ \mathrm{kg} /$ day. Waterlow \& Wills (1960) estimated the faecal metabolic loss at $0.03 \mathrm{~g} / \mathrm{kg} / \mathrm{day}$, and the endogenous 
urinary loss at about $0.05 \mathrm{~g} / \mathrm{kg} /$ day. If these obligatory losses are subtracted from the intake, it appears that the babies studied by Gopalan were utilizing some $85 \%$ of the available N. For some of our babies the corrected utilization was equally high.

Under such conditions of low intake an apparently small superiority of human milk might be of great significance, because it is of critical importance to achieve the highest possible efficiency of utilization.

\section{SUMMARY}

I. A comparison has been made in seventeen malnourished infants of the absorption and retention of nitrogen and phosphorus from human milk, and from cow's-milk mixtures of equal protein, fat and carbohydrate content.

2. $N$ absorption was not significantly different with the two types of milk. On human milk $49 \%$ of ingested $N$ was retained, compared with $41 \%$ on the cow's-milk mixtures. This difference was significant.

3. On both types of milk $70 \%$ of ingested $P$ was absorbed. The $P$ content of human milk is only half that of the cow's-milk mixtures. The percentage retention of $P$ was therefore greater on human milk, but the absolute amounts of $\mathrm{P}$ retained were the same on the two milks.

4. It is suggested that although the difference in $\mathrm{N}$ retention was not very great, it may be of biological significance to infants living on marginal protein intakes, for whom it is of critical importance to achieve the highest possible efficiency of $\mathrm{N}$ utilization.

\section{REFERENCES}

Barness, L. A., Baker, D., Guilbert, P., Torres, F. E. \& György, P. (1957). F. Pediat. 5r, 29.

Bennich, H., Johansson, B. \& Mellander, O. (1957). Acta Soc. med. upsalien, 62, 67.

Block, R. J. \& Bolling, D. (1946). Arch. Biochem. ro, 359.

Cornely, D. A., Barness, L. A. \& György, P. (1957). F. Pediat. 51, 40.

Gopalan, C. (1958). F. trop. Pediat. 4, 87 .

Gordon, H. H., Levine, S. Z., Wheatley, M. A. \& Marples, E. (1937). Amer. F. Dis. Child. 54, ro3o. György, P. (1953). Pediatrics, Springfield, II, 98.

Macy, L. G., Kelley, H. \& Sloan, R. (1950). Bull. nat. Res. Coun., Wash., no. I 19.

Waterlow, J. C. \& Wills, V. G. (I960). Brit. F. Nutr. I4, 183.

Wills, V. G. \& Waterlow, J. C. (1958). F. trop. Pediat. 3, 167. 